\title{
Enhanced Palladium Adsorption by Immobilized Pichia pastoris: A Reusable Catalyst for Reduction of 4-Nitrophenol
}

\author{
Liqin Lin*, Xiaolian Jing, Shiping Chen \\ Department of Chemical Engineering and Pharmacy, College of Chemical Engineering and Materials Science, \\ Quanzhou Normal University, Quanzhou, China \\ Email: *liqin-100@163.com, jingxiaolian@foxmail.com, shiping22@163.com
}

How to cite this paper: Lin, L.Q., Jing, X.L. and Chen, S.P. (2020) Enhanced Palladium Adsorption by Immobilized Pichia pastoris: A Reusable Catalyst for Reduction of 4-Nitrophenol. Journal of Materials Science and Chemical Engineering, 8, 91-97. https://doi.org/10.4236/msce.2020.84007

Received: March 3, 2020

Accepted: April 24, 2020

Published: April 27, 2020

\begin{abstract}
In this paper, the immobilized pellets were prepared by embedding Pichia pastoris ( $P$. pastoris) with sodium alginate and used for the recovery of palladium ions in solution. The catalytic activity of immobilized $\mathrm{Pd} / P$. pastoris for 4-nitrophenol (4-NP) was studied. The results showed that the recovery rate of Pd ions could be significantly increased by immobilized cells. The immobilized $\mathrm{Pd} / P$. pastoris pellets showed good catalytic activity for the reduction of 4-NP, and the catalyst remained good catalytic activity even after multiple reuses.
\end{abstract}

\section{Keywords}

Palladium, Recovery, Immobilized, Pichia pastoris

\section{Introduction}

Due to its unique catalytic activity and high stability in selective hydrogenation and selective oxidation reactions, palladium is widely used as a catalyst in automobile exhaust purification, petrochemical industry and petroleum refining [1]. The reserves of palladium on the earth are very limited. The contradiction between the increase of palladium demand and the shortage of palladium resources will become more and more acute with the rapid development of industrial technology. Therefore, it is particularly important to develop effective technologies to make full use of existing resources and to recover palladium from waste.

It is well known that microbial cells can be used as adsorbents for metal ions due to their advantages of extensive sources, non-toxicity, mild reaction conditions and low cost. Different types of microorganisms have been proved to be 
applicable to the recovery of palladium from the solution [2] [3] [4] [5]. However, the amount of metal ions adsorbed by microorganisms is still relatively limited. For some relatively high concentration metal solutions, a large amount of biomass is needed, which increases the difficulty of subsequent treatment. In recent decades, immobilization biological technology has been applied to biological fermentation, environmental purification, energy production and so on [5] [6] [7]. Microorganisms cells was embed in the carrier skeleton to protect them from the toxicity of pollutants, which not only increases the effective biomass, reduces the cost of cell reconstruction, but also shows good dispersion and recyclability [8] [9]. In this paper, P. pastoris were embed with sodium alginate to recover palladium from the solution, and the immobilized pellets with palladium were used as a supported catalyst for the reduction of 4-np.

\section{Material and Methods}

\subsection{Materials}

P. pastoris was purchased from Invitrogen. Palladium chloride, peptone, yeast extract powder, glucose, 4-nitrophenol, sodium borohydride $\left(\mathrm{NaBH}_{4}\right)$, and other agents used in the experiment were purchased from Sinopharm Chemical Reagent Co., Ltd. All the chemicals and reagents were used as received without further purification.

\subsection{Biosorption of Pd(II)}

$P$. pastoris were incubated in the medium containing $10 \mathrm{~g} / \mathrm{L}$ yeast extract, $20 \mathrm{~g} / \mathrm{L}$ peptone, and $20 \mathrm{~g} / \mathrm{L}$ glucose at $30^{\circ} \mathrm{C}$ for $48 \mathrm{~h}$. The yeast was harvested by centrifugation at $3000 \mathrm{rpm}$ for $10 \mathrm{~min}$, rinsed twice with deionized water and freeze-dried. The biosorption experiments were performed by adding $P$. pastoris biomass into $100 \mathrm{~mL}$ of $\mathrm{Pd}(\mathrm{II})$ solutions, which was stirred on a shaker at a rotation rate of $150 \mathrm{rpm}$ in the dark at $30^{\circ} \mathrm{C}$. Samples were taken at regular intervals for analysis.

$0.7 \mathrm{~g}$ sodium alginate was dissolved in $50 \mathrm{~mL}$ of distilled water. Afterwards, it was mixed with the prepared $P$. pastoris yeast solution $(10 \mathrm{~mL})$ and released dropwise into $0.05 \mathrm{M} \mathrm{CaCl}_{2}$ solution. The beads were soaked in $\mathrm{CaCl}_{2}$ solution for $1 \mathrm{~h}$ to improve their mechanical strength and washed three times with distilled water to remove excess calcium ions. The biosorption experiments were conducted and samples were taken at regular intervals for analysis.

\subsection{Characterization}

Scanning electron microscopy (SEM; ZEISS Sigma) was applied to analyze the morphology of immobilized $\mathrm{Pd} / P$. pastoris. $\mathrm{Pd}$ concentration was determined using atomic absorption spectroscopy (AAS, TAS990).

\subsection{Catalytic Reduction of 4-NP}

In a typical reduction experiment, $0.1 \mathrm{~mL}$ of $1 \mathrm{mmol} / \mathrm{L} 4-\mathrm{NP}$ was mixed with 2.9 
$\mathrm{mL}$ of $0.1 \mathrm{~mol} / \mathrm{L} \mathrm{NaBH}_{4}$ solution in a quartz colorimetric dish and then three immobilized $\mathrm{Pd} / P$. pastoris pellets were added. The UV-Vis absorption spectra at a given time were taken. The conversion ratio of 4-NP can be calculated by the followed equation:

$$
\text { Conversion }(\%)=\left(1-A_{t} / A_{0}\right) * 100 \text {. }
$$

After the reaction, the pellets were recycled from the solution by tweezers and rinsed with distilled water. The reuse catalytic activity was studied for several runs.

\section{Results and Discussion}

There were abundant functional groups in microbial cells surface such as sulfur, hydroxyl, carboxyl and other groups, which can be used as adsorbent to recover metal ion in solution through surface complexation, ion exchange, electrostatic attraction and other effects. Herein, P. pastoris was used as an adsorbent to study the effect of different initial palladium concentrations of on the adsorption efficiency. As shown in Figure 1(A), the adsorption efficiency of palladium increases with the extension of reaction time. When the reaction time was $60 \mathrm{~min}$, a dynamic equilibrium was reached and the adsorption rate of palladium was only $64.5 \%$. Extending reaction time little influence has little effect on the Pd uptake. However, the adsorption ratio of palladium decreased with the increase of initial palladium concentration. Therefore, it is necessary to take some measures to improve the recovery efficiency of palladium.

In order to enhance the recovery efficiency of palladium, the immobilized pellets were prepared by embedding $P$. pastoris with sodium alginate. It can improve the dispersion and stability of microbe cell and avoid the loss of microbe in the reaction process. Moreover, the recycle process is facile. Figure $2(\mathrm{~A})$ and Figure 2(B) are photos of the as-prepared immobilized pellets. The microspheres with a diameter of $3 \mathrm{~mm}$ are uniform and have good elasticity. The SEM
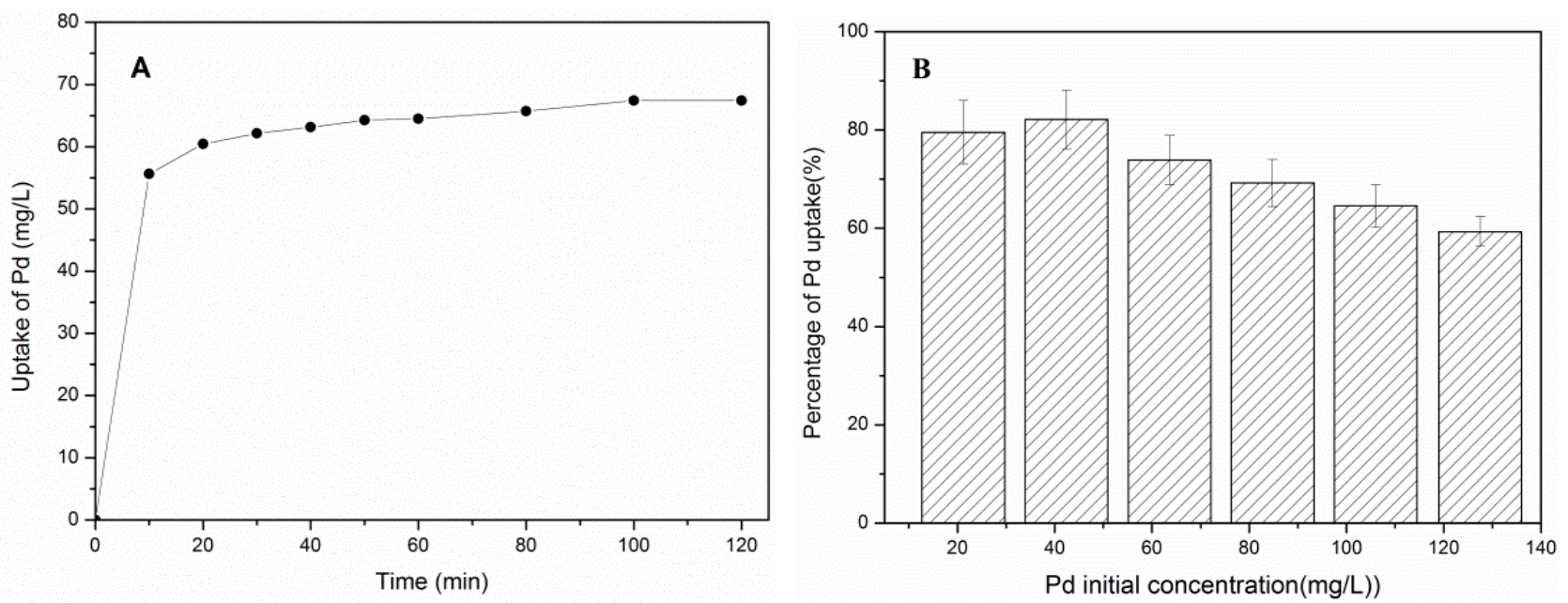

Figure 1. (A) Percentages of Au uptaken by P. pastoris versus time (P. pastoris. $4 \mathrm{~g} / \mathrm{L} ; \mathrm{Pd}$ (II): $\left.106 \mathrm{mg} / \mathrm{L} ; 30^{\circ} \mathrm{C}\right)$; (B) Effect of Pd initial concentration on the percentage of Pd uptake by $P$. pastoris (P. pastoris. $4 \mathrm{~g} / \mathrm{L} ; 30^{\circ} \mathrm{C} ; 120 \mathrm{~min}$ ). 


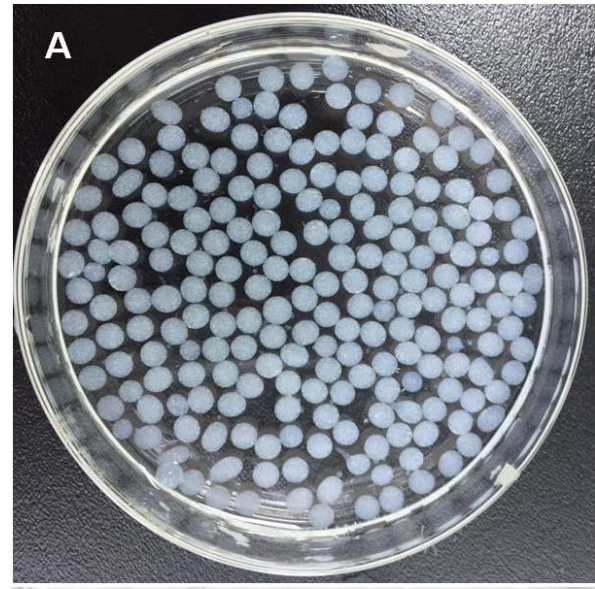

C

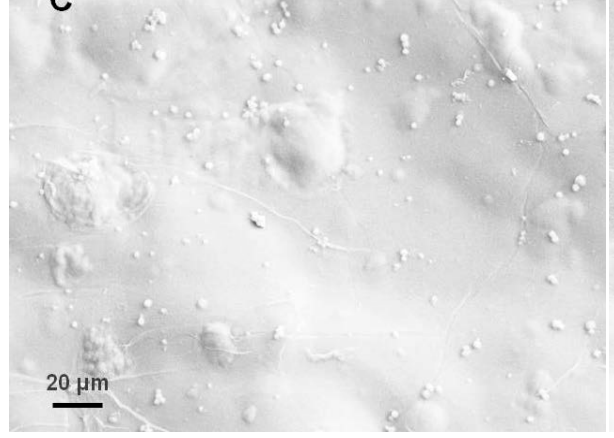

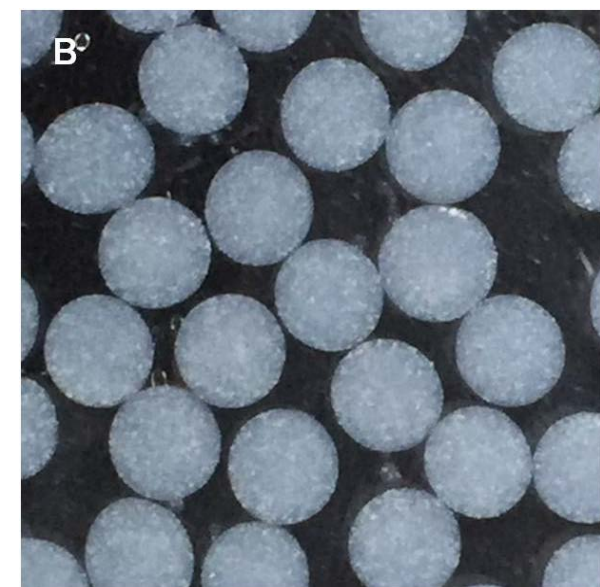

D

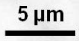

Figure 2. ((A), (B)) Photos and SEM images ((C), (D)) of immobilized P. pastoris cells.

images in Figure 2(C) and Figure 2(D) shows that $P$. pastoris evenly dispersed in the calcium alginate gel beads formed by the cross-linking reaction between sodium alginate and calcium divalent ions.

The immobilized pellets were used to adsorb palladium in the solution and the effects of different $P$. pastoris loads on the palladium adsorption were studied. As shown in Figure 3, even when $P$. pastoris loading capacity was 1 g, almost all palladium ions in the solution were absorbed at $2 \mathrm{~h}$, indicating that the immobilization technique can significantly improve the recovery of palladium in solution.

4-NP is a kind of common organic pollutant in petroleum, medicine, chemical industry and other industrial wastewater. It is highly toxic and difficult to be biodegraded. Direct discharge will cause great harm to both biology and environment. It has been reported that 4-NP can be converted to p-aminophenol (4-AP) by sodium borohydride in the presence of catalyst [10] [11]. Herein, the immobilized pellets adsorbed with palladium (IPP) were served as heterogeneous catalyst for the reduction of 4-NP. As shown in Figure 4, the absorption peak at $400 \mathrm{~nm}$ gradually weakened with the extension of reaction time, indicating that 4-np was constantly converted to 4-AP. The conversion rate was nearly $85 \%$ at 10 minutes.

Although the conversion rate and conversion rate were decreased compared with the $\mathrm{Pd} / P$. pastoris catalyst we reported previously [10], the IPP catalyst 


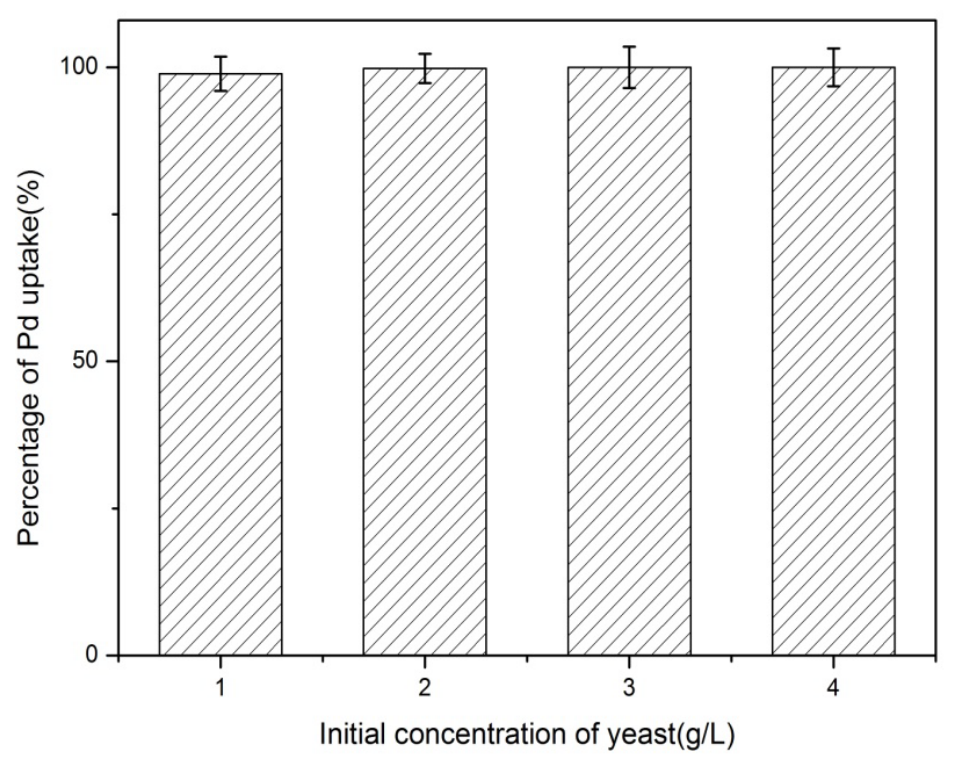

Figure 3. Effect of biomass concentration on the percentage of Pd uptake by immobilized P. pastoris ( $\mathrm{Pd}(\mathrm{II}): 106 \mathrm{mg} / \mathrm{L}, 30^{\circ} \mathrm{C}, 120 \mathrm{~min}$ )

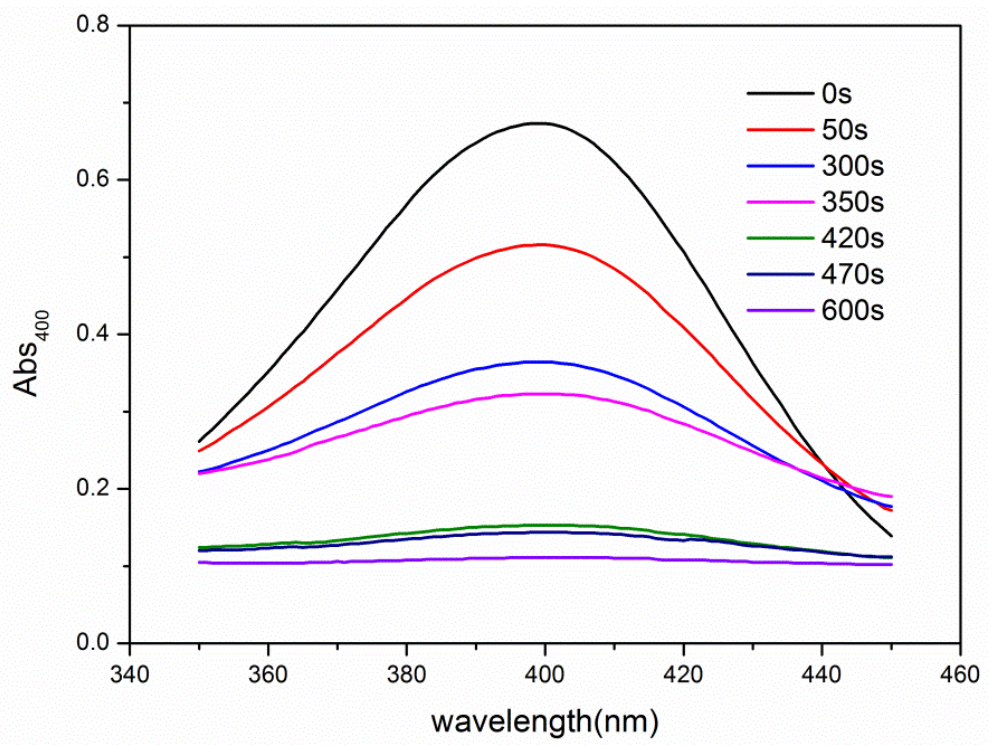

Figure 4. Time-dependent UV-visible absorption spectra for the reduction of 4-NP with immobilized $\mathrm{Pd} / P$. pastoris.

used less at one time and the catalyst could be easily recycled, thereby reducing loss of catalyst during the reaction process. Figure 5 shows the activity of different batches of recovered catalysts. The catalyst activity after the second recovery has been improved compared with the first recovery. That's probably because sodium borohydride further reduced $\mathrm{Pd}(\mathrm{II})$ adsorbed in the pellets to $\mathrm{Pd}(0)$ during the reaction process, increasing the proportion of $\operatorname{Pd}(0)$ in the pellets. Due to the double protection of the functional group on the surface of bacteria and calcium alginate gel, the active component Pd is not easy to agglomerate, leading to the good catalytic activity after repeated recovery. 


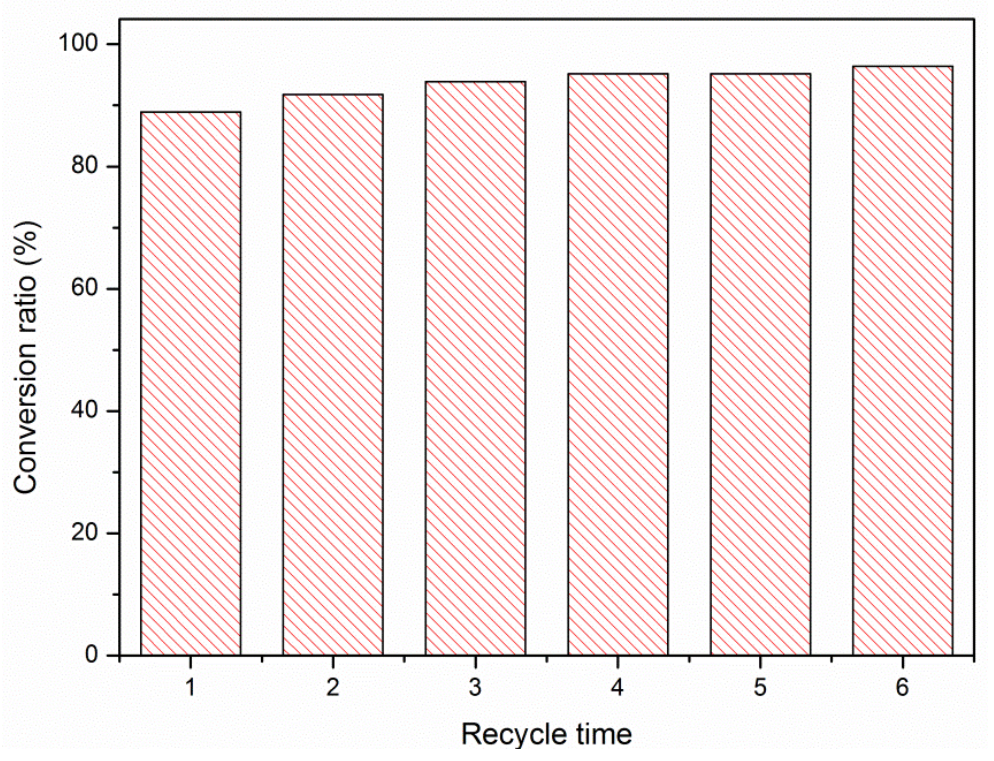

Figure 5. Recycling activities of Pd/IPPC for the catalytic reduction of 4-NP.

\section{Summary}

Compared with $P$. pastoris cells, IPPC pellets can significantly improved the recovery of Pd ions in solution. The immobilized pellets after Pd adsorption were not only easy to recover, but also exhibited good catalytic activity towards the reduction of 4-NP. This may be attributed to the double protection of the abundant functional groups on the cell surface and the calcium alginate gel framework, keeping the good dispersion of PCC and preventing the aggregation of the active components.

\section{Acknowledgements}

We gratefully acknowledge the Natural Science Foundation of Fujian Province (2016J05044).

\section{Conflicts of Interest}

The authors declare no conflicts of interest regarding the publication of this paper.

\section{References}

[1] Yin, L. and Liebscher, J. (2007) Carbon-Carbon Coupling Reactions Catalyzed by Heterogeneous Palladium Catalysts. Chem. Rev., 107, 133-173. https://doi.org/10.1021/cr0505674

[2] Martins, M., Assuncao, A., Martins, H., Matos, A.P. and Costa, M.C. (2013) Palladium Recovery as Nanoparticles by an Anaerobic Bacterial Community. J. Chem. Technol. Biotechnol, 88, 2039-2045. https://doi.org/10.1002/jctb.4064

[3] Hunathai, K., Inoue, K., Ohto, K., Kawakita, H., Kurata, M., Atsumi, K., Fukuda, H. and Alam, S. (2013) Adsorptive Recovery of Palladium(II) and Platinum(IV) on the Chemically Modified-Microalgal Residue. Solvent Extr. Ion Exch, 31, 320-334. 
https://doi.org/10.1002/jctb.4064

[4] Godlewska-Żyłkiewicz, B., Sawicka, S. and Karpińska, J. (2019) Removal of Platinum and Palladium from Wastewater by Means of Biosorption on Fungi Aspergillus sp. and Yeast Saccharomyces sp. Water, 11, 1522. https://doi.org/10.3390/w11071522

[5] Xu, H., Tan, L., Cui, H., Xu, M.Y., Xiao, Y., Wu, H.Y., Dong, H.G., Liu, X.X., Qiu, G.Z. and Xie, J.P. (2018) Characterization of Pd(II) Biosorption in Aqueous Solution by Shewanella oneidensis MR-1. J. Mol. Liq, 255, 333-340. https://doi.org/10.1016/j.molliq.2018.01.168

[6] Ahmad, A., Bhat, A.H. and Buang, A. (2018) Biosorption of Transition Metals by Freely Suspended and Ca-Alginate Immobilised with Chlorella vulgaris. Kinetic and Equilibrium Modeling. J. Cleaner Prod, 171, 1361-1375. https://doi.org/10.1016/j.jclepro.2017.09.252

[7] Xie, J.P., Feng, N.N., Wang, R.H., Guo, Z.W., Dong, H.G., Cui, H., Wu, H.Y., Qiu, G.Z. and Liu, X.X. (2020) A Reusable Biosorbent Using Ca-Alginate Immobilized Providencia vermicola for Pd(II) Recovery from Acidic Solution. Water Air Soil Pollut, 231, 36. https://doi.org/10.1007/s11270-020-4399-Z

[8] Choi, H.A., Park, H.N. and Won, S.W. (2017) A Reusable Adsorbent Polyethylenimine/Polyvinyl Chloride Crosslinked Fiber for Pd(II) Recovery from Acidic Solutions. J. Environ. Manage, 204, 200-206.

[9] Cho, C.-W., Kang, S.B., Kim, S., Yun, Y.-S. and Won, S.W. (2016) Reusable Polyethylenimine-Coated Polysulfone/Bacterial Biomass Composite Fiber Biosorbent for Recovery of Pd(II) from Acidic Solutions. Chem. Eng. J, 302, 545-551. https://doi.org/10.1016/j.cej.2016.05.091

[10] Chen, H.M., Huang, D.P., Lin, L.Q., Odoom-Wubah, T., Huang, J.L., Sun, D.H. and Li, Q.B. (2014) Facile Fabrication of Pd Nanoparticle/Pichia pastoris Catalysts through Adsorption-Reduction Method: A Study into Effect of Chemical Pretreatment. J. Colloid Interface Sci, 433, 204-210. https://doi.org/10.1016/j.jcis.2014.07.038

[11] Song, L., Shu, L., Wang, Y.Q., Zhang, X.F., Wang, Z.G., Feng, Y. and Yao, J.F. (2020) Metal Nanoparticle-Embedded Bacterial Cellulose Aerogels via Swelling-Induced Adsorption for Nitrophenol Reduction. Int. J. Biol. Macromol, 143, 922-927. https://doi.org/10.1016/j.ijbiomac.2019.09.152 